\title{
Neuron and Sensory Epithelial Cell Fate Is Sequentially Determined by Notch Signaling in Zebrafish Lateral Line Development
}

\author{
Takamasa Mizoguchi, ${ }^{1,2}$ Satoshi Togawa, ${ }^{1}$ Koichi Kawakami, ${ }^{3}$ and Motoyuki Itoh ${ }^{1,2}$ \\ ${ }^{1}$ Division of Biological Science, Graduate School of Science and ${ }^{2}$ Institute for Advanced Research, Nagoya University, Nagoya, Aichi 464-8602, Japan, \\ and ${ }^{3}$ Division of Molecular and Developmental Biology, National Institute of Genetics, Mishima, Shizuoka 411-8540, Japan
}

Sensory systems are specialized to recognize environmental changes. Sensory organs are complex structures composed of different cell types, including neurons and sensory receptor cells, and how these organs are generated is an important question in developmental neurobiology.

The posterior lateral line (pLL) is a simple sensory system in fish and amphibians that detects changes in water motion. It consists of neurons and sensory receptor hair cells, both of which are derived from the cranial ectoderm preplacodal region. However, it is not clearly understood how neurons and the sensory epithelium develop separately from the same preplacodal progenitors.

We found that the numbers of posterior lateral line ganglion (pLLG) neurons, which are marked by neurod expression, increased in embryos with reduced Notch activity, but the forced activation of Notch reduced their number, suggesting that Notch-mediated lateral inhibition regulates the pLLG cell fate in zebrafish. By fate-mapping analysis, we found that cells adjacent to the pLLG neurons in the pre-pLL placodal region gave rise to the anterior part of the pLL primordium (i.e., sensory epithelial progenitor cells), and that the choice of cell fate between pLLG neuron or pLL primordium was regulated by Notch signaling. Since Notch signaling also affects hair cell fate determination at a later stage, our study suggests that Notch signaling has dual, time-dependent roles in specifying multiple cell types during pLL development.

\section{Introduction}

Sensory systems are composed of multiple cell types, including sensory receptor cells and neurons. Sensory receptor cells connect to sensory neurons to transmit environmental information to the brain. Many sensory organs, including olfactory epithelium and inner ear, are derived from ectodermal placodes in the vertebrate cranial region. How such complex structures are formed remains a fundamental question in developmental neurobiology.

The lateral line is an anamniote mechanosensory organ that detects local water flow; it is closely related to the amniote auditory system. The lateral line system consists of mechanoreceptive

\footnotetext{
Received Aug. 2, 2011; revised Aug. 31, 2011; accepted Sept. 2, 2011

Author contributions: T.M. and M.I. designed research; T.M. and S.T. performed research; K.K. contributed unpublished reagents/analytic tools; T.M., S.T., and M.I. analyzed data; T.M. and M.I. wrote the paper.

This research was supported by a Grant-in-Aid for Japan Society for the Promotion of Science Fellows (T.M.), the Astellas Foundation for Research on Metabolic Disorders (M.I.), the Takeda Science Foundation (M.I.), the Program for Improvement of Research Environment for Young Researchers from Special Coordination Funds for Promoting Science and Technology (M.I.), which was commissioned by Ministry of Education, Culture, Sports, Science, and Technology in Japan, and Grants-in-Aid for Scientific Research programs in Japan (M.I.). We thank Dr. Verkhusha and Dr. Vladimir for providing plasmids, the Zebrafish National BioResource Project in Japan for providing zebrafish strains, Dr. Nechiporuk for the $T g$ (neurod:EGFP) strain, Dr. Gilmour for the $T g($ (IdnB:lynGFP) strain, T. Satoh for helpful discussions, and M. Kajikawa, H. Matsuo, and R. Inaba for technical assistance.

The authors declare no competing financial interests.

Correspondence should be addressed to Motoyuki Itoh, Division of Biological Science, Graduate School of Science, Nagoya University, Nagoya, Aichi 464-8602, Japan. E-mail: mito@iar.nagoya-u.ac.jp.

DOI:10.1523/JNEUROSCI.3948-11.2011

Copyright $\odot 2011$ the authors $\quad 0270-6474 / 11 / 3115522-09 \$ 15.00 / 0$
}

neuromasts distributed over the body's surface. Each neuromast is composed of hair cells (i.e., mechanosensory receptors) and surrounding support cells. The zebrafish posterior lateral line is derived from an ectodermal placode in the cranial region, adjacent to the otic vesicle, that becomes morphologically visible at $\sim 18 \mathrm{~h}$ postfertilization (hpf). This placode consists of an anterior group of cells that become posterior lateral line ganglion (pLLG) neurons and, posterior to them, the posterior lateral line primordium (pLLP), which gives rise to the neuromasts. The primordium migrates caudally, depositing groups of cells at regular intervals; these cells later differentiate into neuromasts. The posterior lateral line (pLL) system has been used to study cell migration and sensory organ development (Ghysen and DamblyChaudière, 2007; Ma and Raible, 2009). However, how ganglion neurons and sensory-hair precursor cells form from the pLL placode is unclear.

Before and at the start of placode formation, several genes are expressed in the pre-pLL region. six and eya family genes are expressed in all placode precursors (Schlosser, 2006). Although their functions in the pLL system have not been fully investigated, they are involved in otic placode formation (Zheng et al., 2003; Zou et al., 2004; Kozlowski et al., 2005). The bHLH transcription factors neurog1 and neurod begin to be expressed in PLLG neurons before the pLL placode appears. Loss-of-function analysis revealed that neurogl is involved in pLLG but not neuromast hair cell development (Andermann et al., 2002). Therefore, segregation of the neuronal and sensory epithelial lineages occurs before 
the morphological appearance of the pLL placode, although how segregation is achieved remains uncertain.

The Notch-signaling pathway is highly conserved among species and regulates cell fates in a variety of tissues (ArtavanisTsakonas et al., 1999). Notch family proteins are activated by the ligands Delta and Jagged, whose functions are modulated by the ubiquitin ligase Mib (Itoh et al., 2003; Koo et al., 2005; Lai et al., 2005). In Drosophila, Notch signaling regulates distinct cell fates sequentially during sensory organ precursor development in the peripheral nervous system (PNS) (Guo et al., 1996; Zeng et al., 1998). However, in vertebrate sensory organ development, the sequence of Notch signaling functions has not been fully examined.

Here, we examined Notch signaling in zebrafish pLL development and found a previously uncharacterized early role in the fate determination between neuron and sensory epithelial cell progenitors. Since Notch signaling also plays a later role in hair cell fate determination, this finding indicates that Notch signaling has time-dependent dual roles during pLL development.

\section{Materials and Methods}

Fish maintenance and mutants. Zebrafish were raised and maintained under standard conditions. The mib ta52b mutant was described previously (Itoh et al., 2003). The deltaA mutant was newly identified from Tol2 insertion screening by the Kawakami Group (National Institute of Genetics, Mishima, Japan). The Tol2 trap vector is inserted into the first exon of the deltaA gene; a detailed analysis of this mutant line will be reported elsewhere. The Tg(neurod:EGFP) (Obholzer et al., 2008) and $\operatorname{Tg}(\operatorname{cldnB}: \operatorname{lyn} G F P)$ (Haas and Gilmour, 2006) fish strains were obtained from Dr. Nechiporuk (Oregon Health and Science University, Portland, OR) and Dr. Gilmour (European Molecular Biology Laboratory, Heidelberg, Germany), respectively. Males and females of these strains were mated to generate embryos.

Heat shock experiments. For Notch gain-of-function analyses, embryos were obtained from matings between $T g$ (UAS:myc-Notchla-intra) and $\operatorname{Tg}$ (hsp70:Gal4) carriers (Scheer et al., 2002). NotchlaICD (ICD, intracellular domain) expression was induced by heat shock at $40^{\circ} \mathrm{C}$ for 30 $\mathrm{min}$ at the 5 somite stage (ss), after which the embryos were incubated at $28.5^{\circ} \mathrm{C}$ until fixation. After in situ hybridization, the embryos were digested by proteinase $\mathrm{K}$ and genotyped by PCR, as previously described (Scheer et al., 2002).

Whole-mount in situ hybridization and antibody staining. Wholemount in situ hybridization was performed as described previously (Yamamoto et al., 2010). Previously published probes were used for neurod (Korzh et al., 1998), neurog1 (Blader et al., 1997), deltaA, deltaB, deltaD (Haddon et al., 1998a), Notchla (Bierkamp and Campos-Ortega, 1993), notch3 (Itoh and Chitnis, 2001), six1b (Bessarab et al., 2004), and atohla (Kim et al., 1997). Whole-mount antibody staining was performed using the following antibodies. AntiGFP antibodies were purchased (Living Colors A.v. Peptide Antibody, Clontech). A rabbit polyclonal CldnB antibody was generated against peptides CPEKSDSGKYTAKY (aa 185-198) and CATPRSEASAPSGKNFV (aa 200215) of zebrafish CldnB. Alexa Fluor 488 anti-mouse IgG or Alexa Fluor 594 anti-rabbit IgG (Invitrogen) were used as the secondary antibodies. For double staining of in situ hybridization and GFP, in situ hybridization was performed first, followed by anti-GFP staining.

Morpholino antisense oligonucleotide injection. Morpholino oligonucleotide (MO) sequences (Gene Tools) and the dose injected were as follows: deltaB MO, 5'-GTTCCTTACCAACTGCAACAGCGA-3', 5 ng; notch1aATG MO, notch1aUTR MO, notch3ATG MO, and notch3UTR MO, as described previously, $2 \mathrm{ng}$ (Tsutsumi and Itoh, 2007); deltaD MO, 5 ng (Holley et al., 2002); and rbpja/b MO, 5 ng (Sieger et al., 2003).

A standard control MO from Gene Tools was used as a control. Each MO was injected into one- to two-cell stage embryos.

Fate mapping and time-lapse analyses. Fate mapping analysis was performed with PATagRFP (Subach et al., 2010). To synthesize PATagRFP mRNA, PATagRFP was subcloned into a pCS2 + SN vector with EcoRI
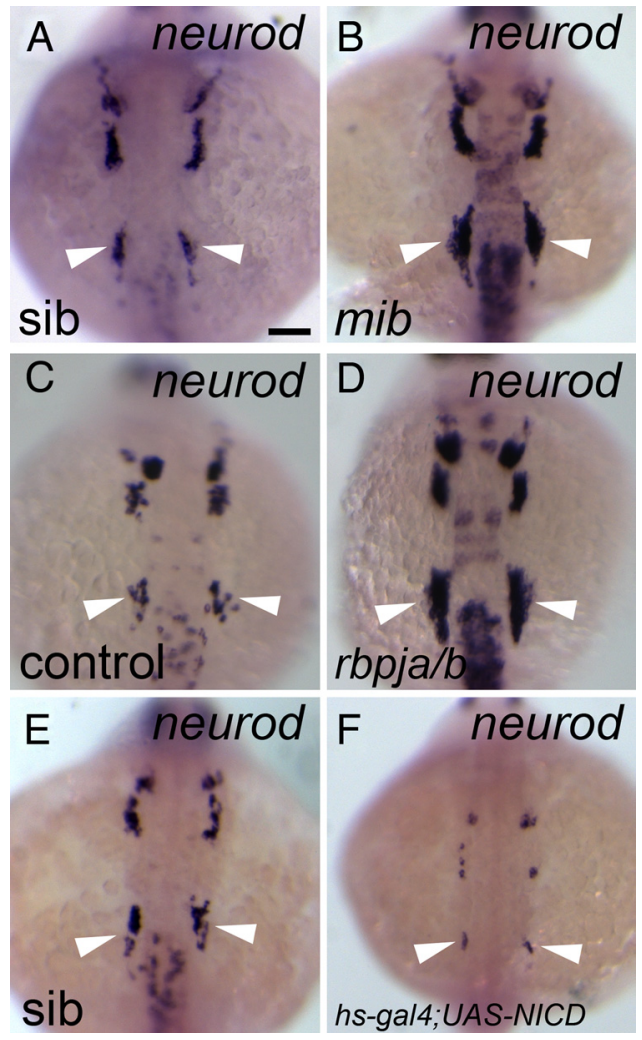

Figure 1. Notch signaling regulates $\mathrm{pLLG}$ cell development. $\boldsymbol{A}-\boldsymbol{F}$, neurod expression at $14 \mathrm{ss}$. Compared to sibling controls $(\boldsymbol{A})$ or control MO-injected embryos ( $\boldsymbol{C}$, the number of $\mathrm{pLLG}$ cells was increased in the mib $(\boldsymbol{B})$ or rbpja/b M0-injected embryos (D). The number of pLLG cells was decreased in the hs-gal4;UAS-NICD embryos $(\boldsymbol{F})$ compared to sibling controls $(\boldsymbol{E})$. White arrowheads indicate pLLG cells. Dorsal views are shown, with anterior to the top. Scale bar, $100 \mu \mathrm{m}$.

Table 1. Defects in Notch signaling increase neurod-expressing pLLG neurons

\begin{tabular}{lrrrr}
\hline & \multicolumn{3}{l}{ neurod expression (\% of embryos) } & \\
\cline { 2 - 4 } Oligo or mutant & + & ++ & +++ & $N$ \\
\hline control & 100 & 0 & 0 & 43 \\
mib & 0 & 0 & 100 & 20 \\
rbpja/b & 0 & 35 & 65 & 26 \\
deltaA & 100 & 0 & 0 & 10 \\
deltaB & 92 & 8 & 0 & 47 \\
deltaD & 92 & 8 & 0 & 39 \\
deltaA, $B$ & 88 & 12 & 0 & 8 \\
deltaA,D & 31 & 69 & 0 & 13 \\
deltaB,D & 88 & 12 & 0 & 25 \\
deltaA, $B, D$ & 14 & 47 & 39 & 28 \\
notch1a & 100 & 0 & 0 & 18 \\
notch3 & 100 & 0 & 0 & 18 \\
notch $1 a, 3$ & 0 & 53 & 47 & 19 \\
\hline
\end{tabular}

Morpholino oligos were injected into wild-type embryos or deltaA mutants, and the phenotype was characterized by the neurod expression at 14 ss.

and NotI sites. Capped mRNA was synthesized from a NarI linearized template using the SP6 mMESSAGE mMACHINE system (Ambion). PATagRFP mRNA (400 pg) was injected into $T g$ (neurod:EGFP); $\operatorname{Tg}(\mathrm{cldnB}$ : lynGFP) or $\operatorname{Tg}$ (neurod:EGFP); $\operatorname{Tg}(\operatorname{cldnB} B \operatorname{lyn} G F P)$; mib embryos at the onecell stage and incubated at $28.5^{\circ} \mathrm{C}$. At $10 \mathrm{ss}$, the embryos were mounted in $1.5 \%$ methylcellulose, photoactivated by ultraviolet rays with a minimum field diaphragm, and incubated again. At 21, 24, or $32 \mathrm{hpf}$, cell fate was analyzed by confocal microscopy (Olympus FV1000 or LSM5 PASCAL, Zeiss). To count the numbers of pLLG and pLLP cells, nuclei were labeled with DAPI after the embryos were stained with anti-GFP and anti-cldnB antibodies. Confocal image stacks were obtained to cover the pLLG (GFP) and pLLP (red; anti-cldnB) regions. The DAPI-stained 

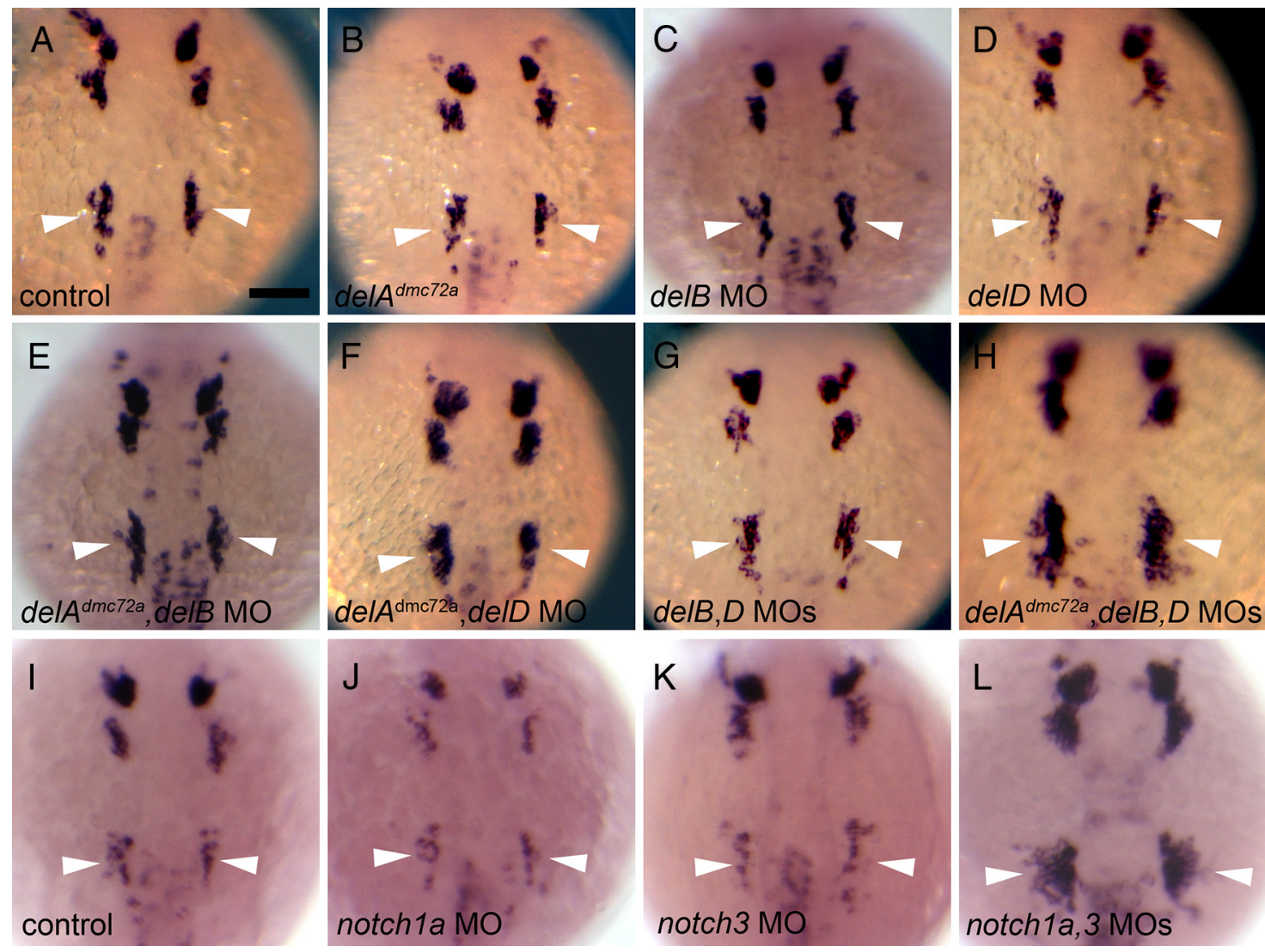

Figure 2. Notch ligands and receptors function redundantly in pLLG development. $\boldsymbol{A}-\boldsymbol{H}$, The expression of neurod in Notch ligand-deficient embryos. Defects in deltaA, $B$, and $D$ genes resulted in the most drastic increase in pLLG neurons, compared to the single or double gene deficiency $(\boldsymbol{H})$. $\boldsymbol{I}-\boldsymbol{L}$, The expression of neurod in notch $1 a$ and/or notch $3 \mathrm{M}$-injected embryos. The double knockdown of notch $1 a$ and notch $3(\boldsymbol{L})$, but not the knockdown of either gene alone $(\boldsymbol{J}, \boldsymbol{K})$, increased the number of neurod-positive pLLG neurons. $\boldsymbol{A}-\boldsymbol{L}$, Dorsal views with the anterior to the left at 14 ss. White arrowheads indicate $\mathrm{pLLG}$ neurons. Scale bar, $100 \mu \mathrm{m}$.

cells were manually marked using FV10-ASW software (Olympus) to count the cells in the images. Time-lapse analysis was performed as described previously (Mizoguchi et al., 2008). Time-lapse movies were generated using ImageJ $(\mathrm{NIH})$.

\section{Results}

Notch signaling regulates the number of pLLG neurons

To determine how pLLG neuronal differentiation occurs, we performed whole-mount in situ hybridization for the proneural marker gene neurod and asked whether Notch signaling were involved in the formation of pLLG neurons. The gene for Mib, a ubiquitin ligase, and that for Rpbj are essential for proper Notch signaling (Itoh et al., 2003; Sieger et al., 2003). Reduced Notch signaling activity in zebrafish has been reported in mib mutants or embryos injected with a morpholino antisense oligo, MO, against rbpja/b (Itoh et al., 2003; Sieger et al., 2003). In mib mutants or $r b p j a / b$ knockdown embryos, the number of pLLG neurons increased, as expected in the absence of Notchmediated lateral inhibition (Fig. 1A-D; Table 1). In contrast, forced transient Notch activation at 5 ss using the hs-gal4;UAS-

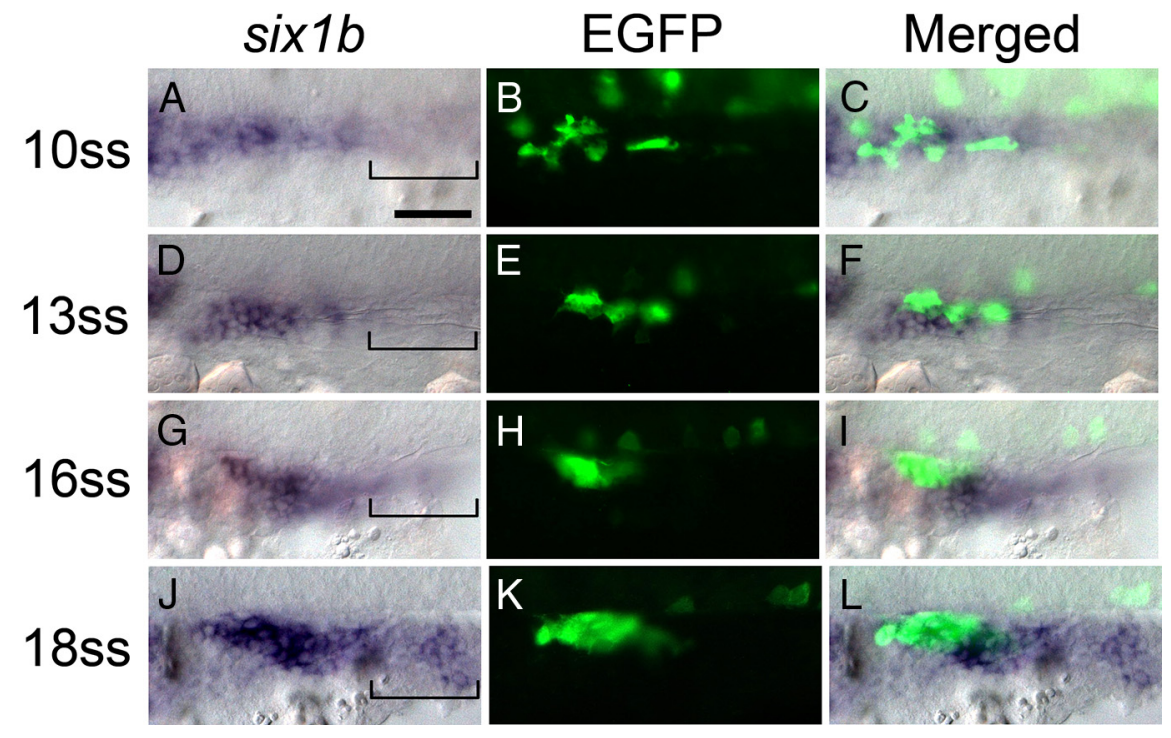

Figure 3. $\quad \mathrm{pLLG}$ neurons arise in the six $1 b$-positive pre-pLL domain, and the six $1 b$ domain expands posteriorly at the late segmentation stage. $\boldsymbol{A}, \boldsymbol{D}, \mathbf{G}, \boldsymbol{J}$, The expression of six $1 \boldsymbol{b}$ at $10,13,16$, and $18 \mathrm{ss}$. $\boldsymbol{B}, \boldsymbol{E}, \boldsymbol{H}, \boldsymbol{K}$, EGFP expressed in $T$ (neurod:EGFP). $\boldsymbol{C}, \boldsymbol{F}, \boldsymbol{I}, \boldsymbol{L}$, Merged views. EGFP-positivepLLGneuronswere generated in thesix $1 b$-positive pre-pLL region $(\boldsymbol{A}-\boldsymbol{C})$ and began to aggregate $(\boldsymbol{D}-\boldsymbol{L})$. The six 1 b expression domain expanded posteriorly after $\mathrm{LL} L \mathrm{G}$ cells aggregated (blackbrackets in $\boldsymbol{A}, \boldsymbol{D}, \mathbf{G}, \boldsymbol{J}) . \boldsymbol{A}-\boldsymbol{L}$,Dorsal views, with anterior to the left. Scale bar, $50 \mu \mathrm{m}$.

NICD system (Scheer et al., 2002) reduced the number of pLLG neurons (Fig. $1 E, F, 100 \%, n=5$ ). These results suggest that Notch signaling is important for regulating the number of newly generated pLLG neurons. 


\section{Delta-mediated Notch signaling is involved in pLLG formation}

Two classes of ligands, Delta and Jagged, activate several Notch receptors. Delta-Notch signaling mediates lateral inhibition in various species, including Drosophila, Xenopus, zebrafish, and mouse (Cabrera, 1990; Chitnis et al., 1995; Haddon et al., 1998a; Daudet and Lewis, 2005; Brooker et al., 2006). We therefore examined whether Notch receptors and their Delta ligands are involved in PLLG development. Loss-of-function analysis using a deltaA mutant line and morpholino antisense oligonucleotides against $\operatorname{deltaB}$ and deltaD revealed that deltaA, B, and $\mathrm{D}$, acted redundantly to restrict pLLG neurogenesis (Fig. $2 \mathrm{~A}-\mathrm{H}$; Table 1). Consistent with this idea, the single knockdown of notchla or notch3 did not significantly affect pLLG neurogenesis, whereas their double knockdown greatly increased it (Fig. 2I-L; Table 1). The most severe phenotype (shown as +++ in Table 1 ) observed with the combined knockdown of delta or notch genes was comparable to that of the mib mutant or $r p b j-a / b$ knockdown embryo. These data agree with the known roles of Mib and $\mathrm{Rbpj}$, which are important for the signaling of multiple Delta and Notch proteins (Oka et al., 1995; de la Pompa et al., 1997; Itoh et al., 2003).

\section{pLLG neurons arise from the six $1 b$-positive preplacodal region}

Various sensory organs composed of neurons and sensory receptor cells are formed from distinct ectodermal placodes. Previous studies showed that the pLLG neurons and pLLP cells, which differentiate into sensory hair and support cells, are already spatially separated into the rostral one-fourth and the caudal threefourths of the preplacodal region, respectively, at $17 \mathrm{hpf}$ (Sarrazin et al., 2010). However, it is not well understood how the pLLG neurons and pLLP (i.e., sensory epithelial cells) are generated within the pLL placode region at earlier stages.

Although the vertebrate preplacodal marker six $1 b$ (Schlosser, 2006) is expressed broadly in the preplacodal ectoderm until the early somite stage (around $11 \mathrm{hpf}$ ), its expression is later confined to sensory placodes, including the zebrafish pLL placode (Bessarab et al., 2004). Since pLLG neurons can first be detected by neurod expression around $10 \mathrm{ss}$ ( $14 \mathrm{hpf}$ ), we next examined the position of neuronal precursors relative to the six $1 b$-positive prepLL domain by double staining for six $1 b$ and neurod using Tg(neurod:EGFP) embryos (Obholzer et al., 2008).

The EGFP-positive pLLG neurons were dispersed within the six $1 b$-expressing domain at 10 ss (Fig. $3 A-C$ ). Later, these neurons migrated rostrally and assembled to form ganglia (Fig. $3 D-L$; Movie 1). They did not divide during our time-lapse observation (14 to $21.5 \mathrm{hpf}$; Movie 1). This is consistent with the observation that pLLG neurons become postmitotic sometime between 8 and 13 hpf (Metcalfe, 1989; Sarrazin et al., 2010). We further found that the six $1 b$ expression domain expanded posteriorly after 18 ss (around 17-18 hpf) (Fig. 3A,D, G,J, black brackets). This posterior six $1 b$ expansion roughly coincided with the morphological appearance of the pLL placode. These data raised the possibility that neurons and sensory epithelial cells are initially intermingled but later segregate spatially.

\section{Non-neuronal cells proximal to the pLLG neurons at 10 ss become the anterior part of the posterior lateral line primordium}

Using fate-mapping techniques, we investigated the possibility that pLLG and pLLP cells arise from the same pre-pLL region (Fig. 4A). We used a $\operatorname{Tg}($ neurod:EGFP); $\operatorname{Tg}(\operatorname{cldnB} B$ :lynGFP) double transgenic line to visualize the pLLG and pLLP (Haas and

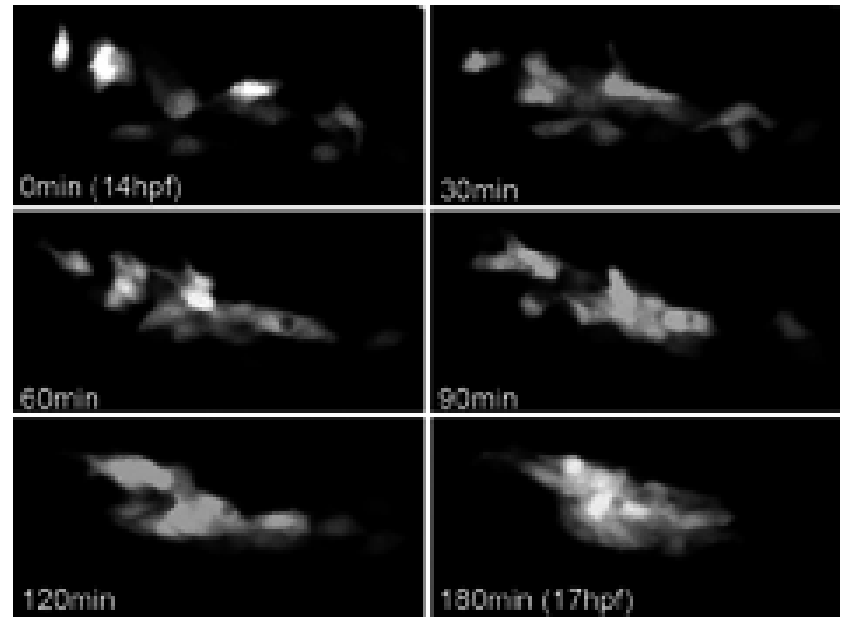

Movie 1. Time-lapse movie of a wild-type $T$ (neurod:EGFP) pLLG from $14 \mathrm{hpf}$ (10 ss) to 17 hpf (16 ss). Z-stack images ( $5 \mu \mathrm{m}$ steps) were acquired every 5 min using an FV-1000 system (Olympus) with a $40 \times$ oil-immersion lens. The movie was generated using ImageJ (NIH). Scattered pLLG neurons aggregated and formed a ganglion.

Gilmour, 2006; Obholzer et al., 2008). We also used PATagRFP, a photoactivatable red fluorescence protein, to label cells locally (Subach et al., 2010). PAtagRFP mRNA was injected into $\mathrm{Tg}($ neurod:EGFP); $T g(\operatorname{cldnB}: \operatorname{lyn} G F P)$ double transgenic embryos, which were raised until $10 \mathrm{ss}$. We then illuminated small regions of the embryos with UV light to photoactivate the protein to red fluorescence. We illuminated cells at different positions near the neurod:GFP-expressing cells (Fig. $4 B$, arrowheads) and followed the fate of the red fluorescent-labeled cells at $24 \mathrm{hpf}$ (Fig. $4 \mathrm{~B}$ ). When we labeled wild-type cells, including neurod:GFP cells, the cells were later found both in the ganglion and the pLLP anterior region (Fig. $4 B$, top panels of sib). Furthermore, when we labeled a few cells in the vicinity of neurod:GFP pLLG, the labeled cells gave rise to anterior pLLP cells (Fig. 4C, top panels of sib). On the other hand, labeled cells caudally adjacent to the neurod:GFPpositive cells later gave rise to a few pLLG neurons and the middle part of the pLLP (Fig. $4 B$, middle panels of sib). Labeled cells further to the posterior gave rise to the posterior part of the pLLP (Fig. $4 B$, bottom panels of sib).

At $32 \mathrm{hpf}$ it could be seen that labeled cells, including the neurod:GFP cells labeled at 10 ss, gave rise to the first and second neuromasts, L1 and L2, and to interneuromast cells between pLLG and L2 (Fig. 4D, magenta arrowheads). This was due to the deposition of neuromast cells migrating from the pLLP, since no labeled cells remained in the pLLP (Fig. 4D). Collectively, these data suggest that the pre-pLL region is already patterned along the anterior-posterior axis at $10 \mathrm{ss}$, and the pLLG and the anterior part of the pLLP cells arise from the same six $1 b$-positive pre-pLL region.

The neuron versus epithelial primordium cell fate is regulated by Notch signaling

Results from the pLLG development and pre-pLL cell-fate mapping analyses raised the possibility that Notch signaling regulates the cell-fate choice between pLLG neurons and pLLP, since Notch signaling is involved in alternative cell fate determination in many tissues. Thus, we performed the same cell fate mapping analysis in mib mutants. The labeled cells, including neurod:GFP cells at 10 ss, gave rise primarily to pLLG neurons and rarely to the anterior part of the pLLP (Fig. $4 B$, top panels of $m i b$ ). When more restricted groups of cells were labeled, almost none gave rise to 
A
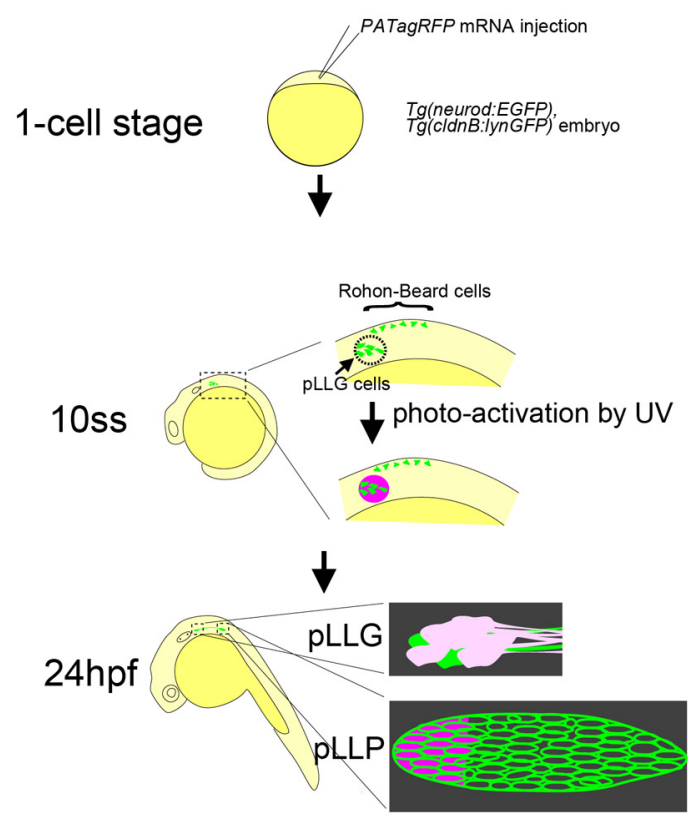

Confocal microscopic observation
B

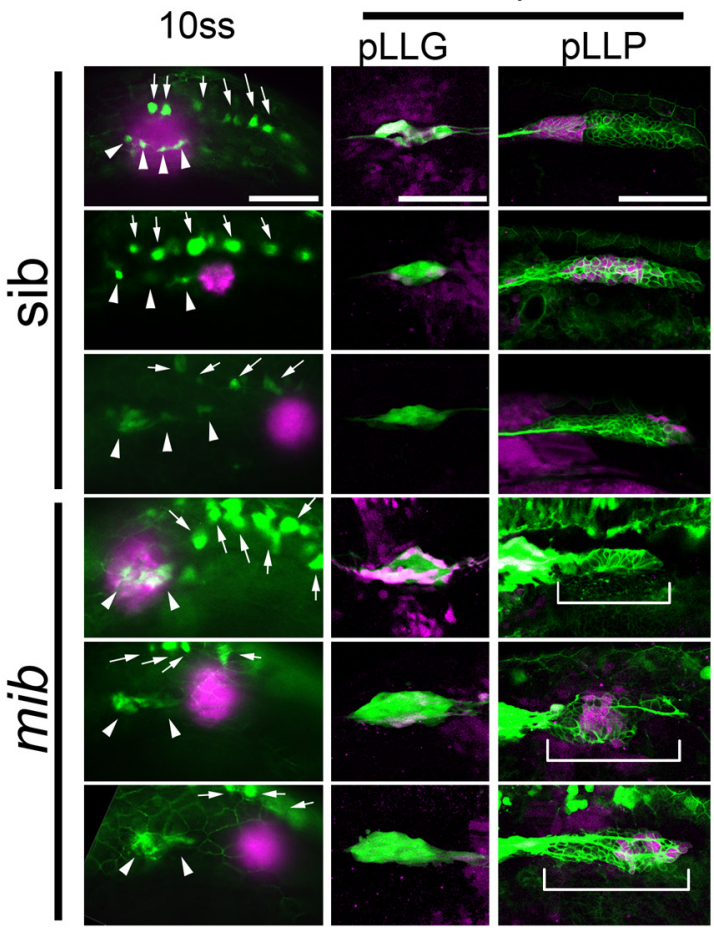

D

10ss
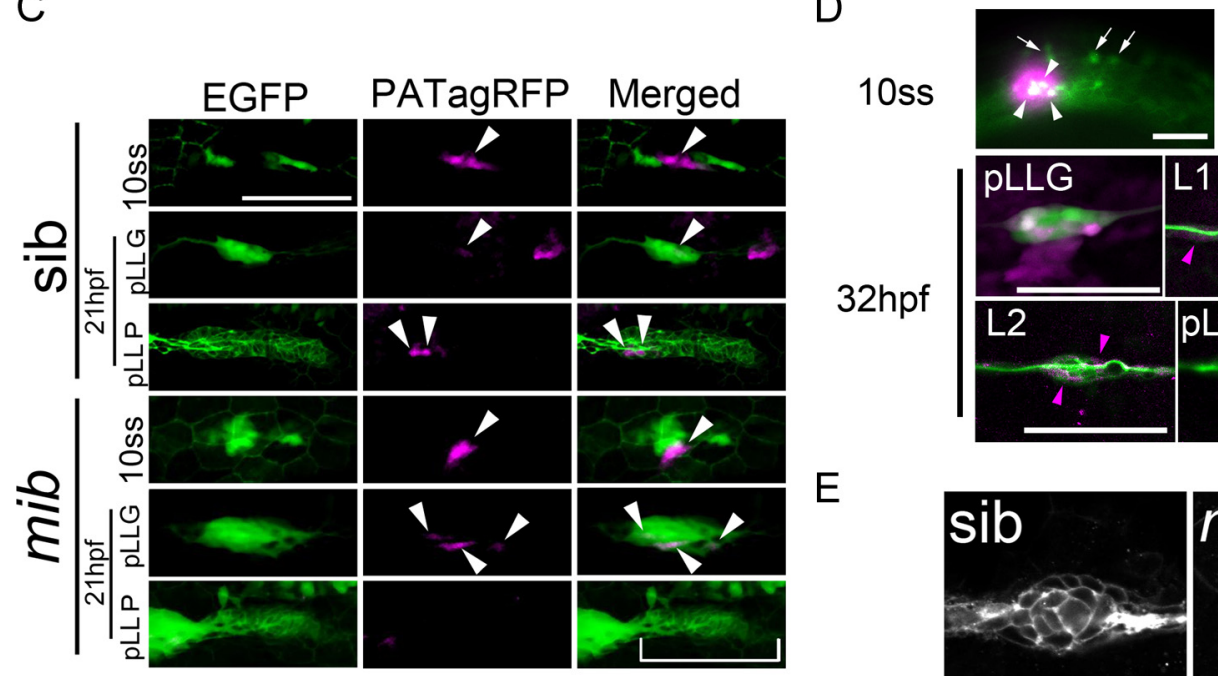

10ss

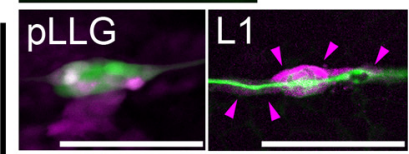

$32 \mathrm{hpf}$

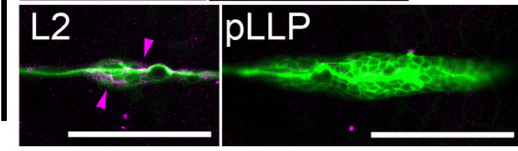

$E$
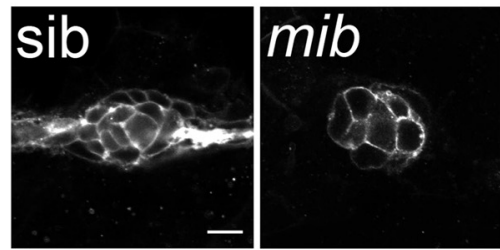

Figure 4. The cell fate of pre-pLL cells is regulated by Notch signaling and is determined at 10 ss along the anterior-posterior axis. $\boldsymbol{A}$, Diagram of the method for fate-mapping analysis. $\boldsymbol{B}$, Fate-mapping analysis in sibling control and mib mutant embryos. After UV activation of cells covering the pLLG (top in sib), proximal to the pLLG (middle in sib), and distal to the pLLG (bottom in sib) at 10 ss (left), the cell fates were analyzed at $24 \mathrm{hpf}$ (middle, pLLG; right, pLLP). The magenta cells are PATagRFP-labeled cells. C, Fate-mapping analysis with fewer cells labeled in the vicinity of the neurod:GFP pLLG. Cells in the vicinity of the pLLG were UV activated (top panels in sib or mib), and the cell fates were analyzed at $21 \mathrm{hpf}$ (middle, pLLG; bottom, pLLP in sib or mib). White arrowheads indicate the PATagRFP-labeled cells. B, C, White brackets indicate the pLLP in mib mutants. D, Fate-mapping analysis at $32 \mathrm{hpf}$. After UV activation of cells covering the pLLG neurons at $10 \mathrm{ss}$, the cell fates were analyzed at $32 \mathrm{hpf}$. Magenta arrowheads indicate PATagRFP-labeled cells. $B, D$, White arrowheads and arrows indicate pLLG neurons and Rohon-Beard cells, respectively. $\boldsymbol{E}$, The number of $L 1$ cells was decreased in the mib mutants. $\boldsymbol{B}-\boldsymbol{E}$, Side views, with anterior to the left. Scale bars, $\boldsymbol{B}, \boldsymbol{C}, 100 \mu \mathrm{m} ; \boldsymbol{D}, 10 \mu \mathrm{m}$.

the anterior pLLP cells $(90 \%, n=10$; Fig. 4C). Labeled cells from posterior regions gave rise to a few pLLG neurons and the anterior-middle part of the pLLP, and cells from even more posterior regions contributed to the posterior part of the pLLP (Fig. 4B, middle and bottom panels of mib).

We also observed a delay in neuromast deposition in the mib mutants. At $32 \mathrm{hpf}, 33 \%$ of the mutants had no deposited neuromasts, $53 \%$ had one, and $13 \%$ had two $(n=15)$. In contrast, at the same stage $88 \%$ of the sibling (sib) control embryos had two deposited neuromasts, and the rest had three $(n=16)$. To avoid artifactual differences in cell number caused by the timing of deposition, we examined the L1 cell number at $27 \mathrm{hpf}$ in sibling controls, when $85 \%$ of the embryos had one deposited neuromast and the rest had none $(n=13)$, and compared it to that in mib mutants at $32 \mathrm{hpf}$ (Fig. 4E). The number of L1 neuromasts that were deposited from the anterior part of the pLLP of the mib embryos was 


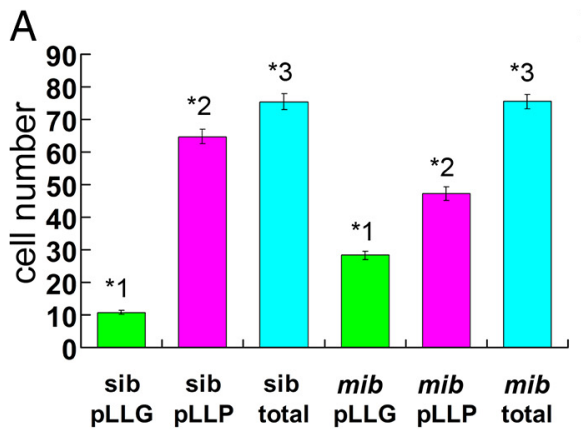

B

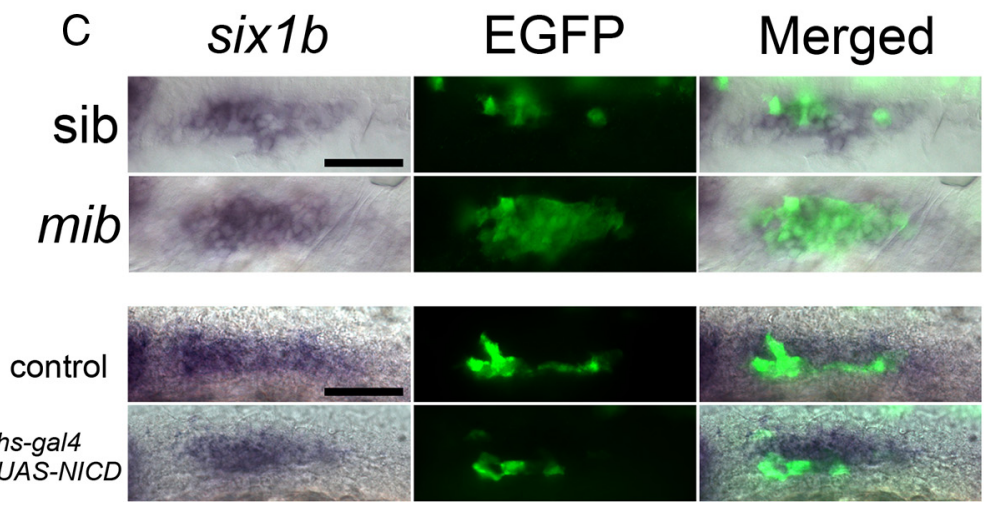

Figure 5. Notch signaling affects the pLLG versus pLLP cell fate. $\boldsymbol{A}$, In mib mutants, the $p L L G$ neurons were increased at the expense of pLLP cells. Cell counts for the pLLG neurons and pLLP cells are given in the $y$-axis. The pLLG neurons increased (green bars) and the pLLP cells decreased (magenta bars), whereas the total number of cells was unchanged (blue bars). Sibling control, $n=16 ;$ mib,$n=17 . B$, In hs-gal4;UAS-NICD embryos, pLLP cells were increased at the expense of pLLG neurons. Cell counts for the pLLG and pLLP cells are given in the $y$-axis. Control (con) embryos, $n=18$; $h$ s-gal4;UAS-NICD embryos, $n=18$. While the pLLG neurons decreased (green bars) and pLLP cells increased (magenta bars), the total cell number was unchanged (blue bars). $\boldsymbol{A}, \boldsymbol{B}$, Error bars represent standard error. ${ }^{*} 1,{ }^{*} 2, * 4$, and ${ }^{*} 5$ indicate statistically significant differences $\left(p<1.0 \mathrm{E}-5\right.$, Student's $t$ test); ${ }^{*} 3$ and ${ }^{*} 6$ indicate no statistically significant difference ( $p>0.05$, Student's $t$ test). $C$, The size of the six $1 b$-positive domain, from which both pLLG and pLLP are generated, was not dramatically changed by altered Notch activity at $14 \mathrm{ss}$. Purple, six $1 b$-positive cells; green, EGFP-positive pLLG cells in Tg(neurod:EGFP). Scale bar, $50 \mu \mathrm{m}$.

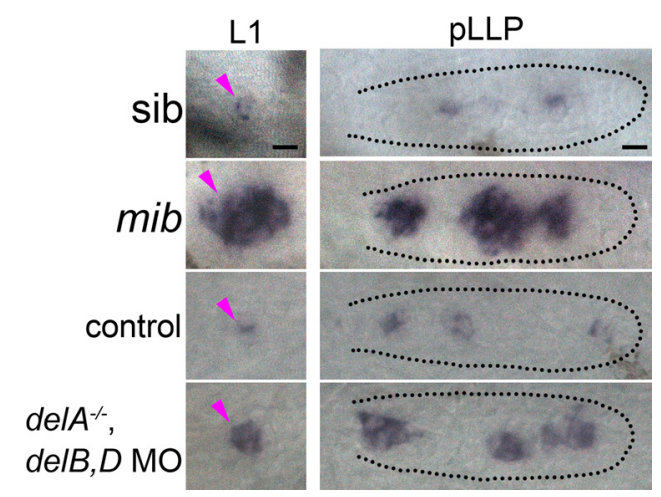

Figure 6. Delta-Notch signaling regulates hair cell fate. Hair cell differentiation at $32 \mathrm{hpf}$ was assessed by atoh $1 a$ expression. The number of hair cells in the L1 neuromast and the pLLP increased in the mib and in the deltaB and D M0-injected deltaA $A^{d m c 72 a}$ mutants. Magenta arrowheads indicate atoh 1a-positive hair cell precursors. Magenta dotted lines outline the pLLP. Shown are lateral views, with anterior to the left. Scale bar, $10 \mu \mathrm{m}$.

smaller than that in sibling controls (Fig. $4 E$; sib: $23.2 \pm 0.9(n=11)$; mib: $11.8 \pm 1.8(n=13)$; L1 cell number, mean \pm SEM; $p<5.0 \mathrm{E}-$ 10). These results indicate that Notch signaling indeed regulates the cell fate choice between pLLG neurons and the pLLP.

To confirm the role of Notch signaling in cell fate choice, rather than in the maintenance or growth of pre-pLL cells, we counted the neurons and primordium cells at $21 \mathrm{hpf}$ in embryos with altered Notch activity. In the mib mutant embryos the number of neurons was increased whereas the number of primordium cells was decreased, compared to sibling controls (Fig. 5A). On the other hand, forced Notch signaling resulted in fewer neurons and an increased number of primordium cells (Fig. 5B). The sum of the two cell populations was not significantly different in each case. To further validate our findings, we examined the ratio of pLLG (neurod) to the pre-pLL domain (six1b) at an earlier stage (14 ss, $16 \mathrm{hpf}$ ), since pLLP marker genes before $21 \mathrm{hpf}$ have not been reported (Fig. 5C). The size of the sixlbpositive domain, from which both pLLG and pLLP are generated, was not dramatically changed by the altered Notch activity (Fig. 5C). In contrast, the number of neurod-positive pLLG cells increased to almost equal the number of six $1 b$-positive cells in the mib mutant, and transient Notch activation reduced the pLLG cell number (Fig. 5C). These findings suggest that Delta-Notch signaling plays an important role in cell fate determination rather than in cell growth in each lineage after their segregation, and that Notch activation favors the primordium fate at the expense of the neuronal fate within the pre-pLL region.

Delta-Notch signaling controls hair cell fate specification in the pLL system

The timing of hair cell specification lags behind the cell fate choice between pLLG and PLLP in pLL development. The HLH factor atohla is a marker of hair cell precursors in the pLL system, and it is expressed in the hair cell lineage after the pLLP separates from the pLLG. We previously proposed a Notch signaling role in the selection of hair cell fate in neuromasts (Itoh and Chitnis, 2001). However, the role of Notch ligands in hair cell fate specification has not been examined. Although the single mutation or knockdown of each gene did not significantly affect the atohla expression in L1 or pLLP, the compound knockdown of the three delta genes increased the number of atohla-expressing hair precursors among the L1 and pLLP cells (Fig. $6 ; 100 \%, n=8$ ). Thus, Delta-Notch signaling regulates cell fate decision sequentially at two different developmental stages in the pLL system.

\section{Discussion}

A model of the pLLG/pLLP-lineage specification in zebrafish From a fate-mapping approach and the analysis of embryos with altered Notch activity, we propose a model for pLL development during early embryogenesis (Fig. 7). At the gastrula stage, the preplacodal domain is initially a broad six $1 b$-positive region (Fig. $7 A)$. Later, the sixlb-positive domain is confined to a smaller domain (the anterior pre-pLL), within which neurod-positive pLLG progenitor cells are selected though lateral inhibition, which is mediated by Delta-Notch signaling (Fig. $7 A, B ; 10 \mathrm{ss}$ ). Neurons and other cells within this domain begin to be segregated spatially (i.e., some neurons assemble to the anterior to form ganglia and others move toward the posterior and give rise to the anterior part of the pLLP). In contrast to the anterior 

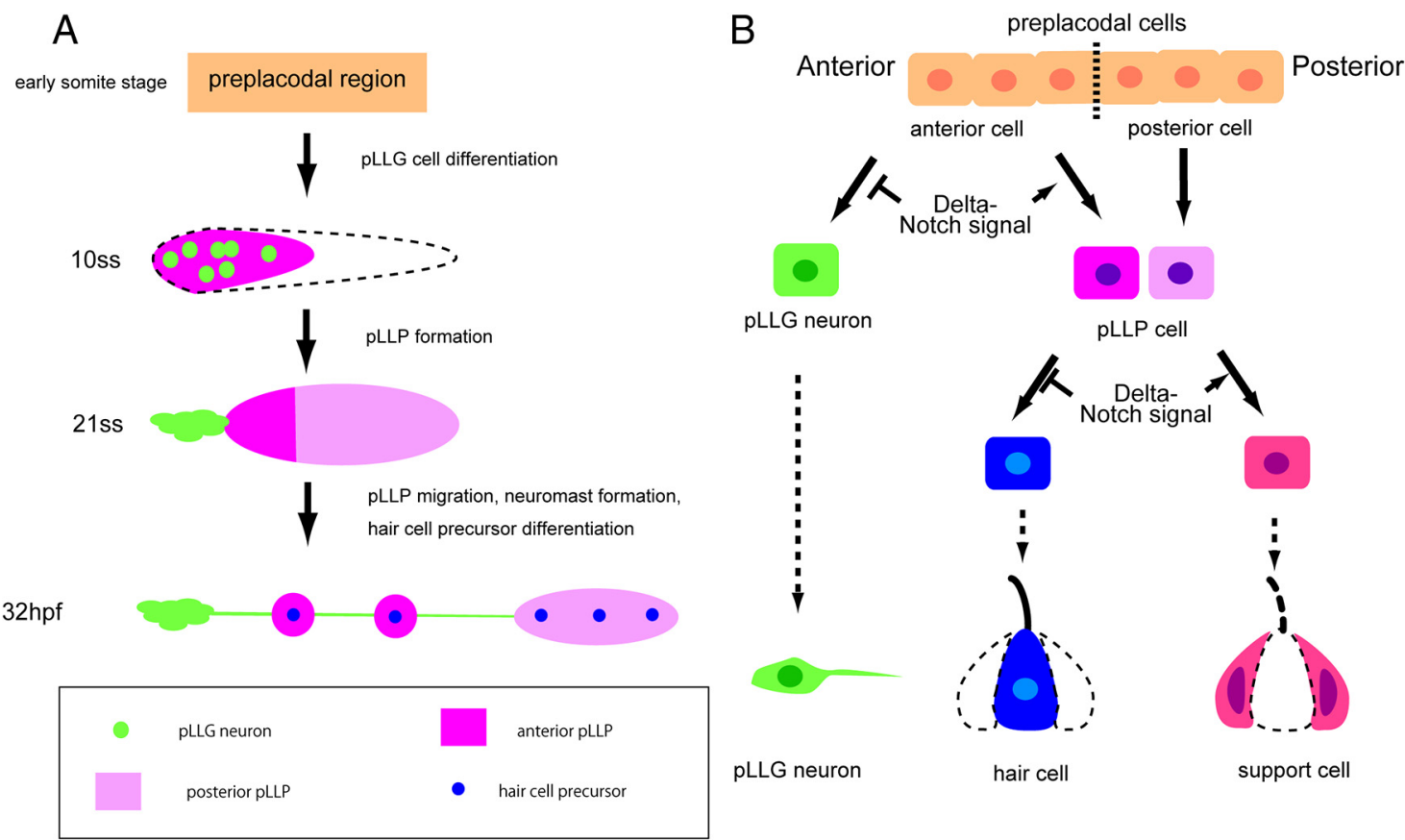

Figure 7. Models for pLLG and pLLP formation and cell-lineage specification regulated by Notch signaling. $A, A$ model for $p L L G$ and pLLP formation. In the early somite stages, six $1 b$ is broadly expressed (orange). At $10 \mathrm{ss}$, six 16 becomes confined to a smaller domain (magenta). At 21 ss, six 16 expression expands to the posterior pre-pLLP (pink). $\boldsymbol{B}$, Notch signaling's time-dependent roles in cell-fate specification in the pLL system.

pre-pLLP, the posterior pre-pLLP domain does not express six $1 b$ at $10 \mathrm{ss}$, but is already fate determined along the anterior-posterior axis. The posterior pre-pLLP gradually acquires six $1 b$ expression and fuses with the anterior pre-pLLP to form a morphologically apparent pLLP at 21 ss (Fig. 7A). The separated pLLP migrates further to the posterior, depositing neuromast precursor cells (Fig. 7A; $32 \mathrm{hpf}$ ). pLLG neurons extend their axons to the neuromasts; each neuromast consists of hair and support cells. Notch signaling also regulates hair cell precursor specification in the pLLP (Fig. 7B).

Collectively, our data indicate that Delta-Notch signaling functions at two steps in posterior lateral line development (Fig. $7 B$ ): first in the pLLG neuron versus anterior pLLP cell fate determination, and then in the hair cell versus support cell fate determination. Thus, Delta-Notch signaling controls the proper posterior lateral line formation spatiotemporally in a stepwise fashion.

\section{pLLG neurogenesis from the preplacodal region}

Neurogenic placodes in the cranial region are initially observed as preplacodal ectoderm expressing six and eya family members (Sahly et al., 1999; Pandur and Moody, 2000; Bessarab et al., 2004; Zou et al., 2004; Schlosser, 2006; Streit, 2007). In the zebrafish posterior lateral line system, six $1 b$ and eyal expression begins in the preplacodal area and continues in pLLG neurons and in the pLLP (Sahly et al., 1999; Bessarab et al., 2004). Our study revealed that neurod-positive pLLG neurons are selected from the six $1 b$ positive preplacodal region through lateral inhibition.

This mechanism is very similar to that leading to the formation of sense organs from proneural fields in Drosophila. In Drosophila, expression of the achaete-scute gene complex members, which encode bHLH factors, is determined by the activities of combinations of prepattern genes and is initially observed relatively uniformly at very low levels (proneural fields) (Cubas et al., 1991; Calleja et al., 2002). By the time of lateral inhibition, this uniform expression has shifted so that a smaller number of cells express the bHLH factors at higher levels, and in the absence of Notch signaling more numerous cells within the proneural fields express high levels of proneural genes (Cubas et al., 1991; Schweisguth and Posakony, 1992). Our results support a Notchmediated lateral inhibition mechanism for neuron selection from the pre-pLL domain, because the number of neurons was altered within the six $1 b$-positive pre-pLL domain (similar to the proneural field) when the Notch activity was changed.

\section{Notch-mediated cell-fate determination affects $\mathrm{L} 1$ neuromast cell deposition}

After fusion of the anterior-pLLP and posterior-pLLP, the pLLP migrates posteriorly and deposits a cluster of cells, known as the neuromast, at regular intervals. A reduction in Notch activity decreases the number of anterior-pLLP cells and of L1 neuromasts, which are derived from the anterior-pLLP cells. This observation was somewhat surprising, as previous studies showed that a reduced pLLP deposits fewer neuromasts of the normal size, rather than smaller neuromasts (Aman et al., 2010; Gamba et al., 2010). These previous studies suggest the existence of a mechanism in which the number of deposited cells is maintained, even when the number of pLLP cells is reduced.

One possible reason for this discrepancy is that this mechanism may not affect L1 deposition. As we found here, the progenitor cells for L1 (in the anterior pLLP) and those for the more posterior neuromasts (L2-L5, in the posterior pLLP) are generated differently. Furthermore, the anterior pLLP makes up only about one-fourth of the entire pLLP, and it is less proliferative (Laguerre et al., 2005). Thus, the effects of experimentally reduced cell proliferation rates on the $\mathrm{L} 1$ progenitors might be more subtle than their effects on the posterior progenitors, which could explain why the number of L1 cells was not significantly affected in the previously reported results. In contrast, in mib mutants the number of anterior pLLP (i.e., future L1, L2) cells is 
selectively reduced compared with that of the posterior pLLP (future L2-L5) cells.

Although we do not know how the L1 deposition is regulated, our results suggest that it does not necessarily depend on the cell number. Another possibility is that Notch signaling is again involved, this time in neuromast deposition. The smaller size of the deposited neuromasts in mib mutants was also observed previously (Matsuda and Chitnis, 2010). Fragmentation of the pLLP in the mib mutants is observed from around $28 \mathrm{hpf}$ and it precedes a loss of $e$-cadherin expression. Although the reason for this early defect in cohesiveness is not known, the reduced cohesiveness may also contribute to the reduced size of L1 neuromast in the mib mutants.

\section{Sequential Delta-Notch signaling in the cell-fate specification of sensory organs: an evolutionarily conserved mechanism} Our results suggest that Delta-Notch signaling influences the cell fates of pLL-lineage cells sequentially: first, in the cell fate choice between pLLG neurons versus anterior-pLLP sensory epithelium, and next in the choice between hair cells versus support cells within the sensory epithelium lineage. Similarly, in flies, the PNS sensory organ lineage is sequentially regulated by Notch signaling to produce distinct daughter cells (Guo et al., 1996; Zeng et al., 1998). However, there are two opposing roles for Notch in hair cell development: its early role functions to increase hair cells, while its later one acts to reduce them.

Notch's later function is well described in ear development in the mouse, chick, and zebrafish (Adam et al., 1998; Haddon et al., 1998b; Kiernan et al., 2005; Brooker et al., 2006). In contrast, Notch signaling's early role in the cell fate decision between neurons versus sensory epithelial cells is not well studied. In the mouse, Brooker et al. (2006) showed that the loss of Delta1 results in excess cochleovestibular ganglion neurons and reduced sensory hair cells in the saccular macula and the utricular macula of the mouse. Together with previous studies showing that the saccular macula and the utricular macula are derived from the region that gives rise to both neurons and sensory hair cells (Satoh and Fekete, 2005; Raft et al., 2007), the data of Brooker et al. (2006) indicate that Delta1-Notch signaling might have an early role in determining the neuron versus sensory epithelium cell fate during mouse inner ear development. In contrast, in zebrafish ear development, an increase in statoacoustic ganglion neurons is accompanied by an increase, not a decrease, in hair cells in the two sensory macula of the Notch-defective mib mutant (Haddon et al., 1998b).

The exact regulatory mechanism for the cell fate decisions in the zebrafish ear is uncertain. Mechanisms other than Notch signaling may, however, determine the neuronal and sensory hair fate in zebrafish ear development since, unlike in the mouse inner ear and zebrafish pLL systems, the neuronal and sensory hair precursors are specified at about the same time in the zebrafish ear (Andermann et al., 2002; Abello and Alsina, 2007; Millimaki et al., 2007; Radosevic et al., 2011). Thus, the regulatory mechanisms in pLL development and mouse ear development may share more similarities than those of zebrafish ear and mouse ear development.

\section{References}

Abello G, Alsina B (2007) Establishment of a proneural field in the inner ear. Int J Dev Biol 51:483-493.

Adam J, Myat A, Le Roux I, Eddison M, Henrique D, Ish-Horowicz D, Lewis J (1998) Cell fate choices and the expression of Notch, Delta and Serrate homologues in the chick inner ear: parallels with Drosophila sense-organ development. Development 125:4645-4654.
Aman A, Nguyen M, Piotrowski T (2010) Wnt/ $\beta$-catenin dependent cell proliferation underlies segmented lateral line morphogenesis. Dev Biol 349:470-482.

Andermann P, Ungos J, Raible DW (2002) Neurogenin1 defines zebrafish cranial sensory ganglia precursors. Dev Biol 251:45-58.

Artavanis-Tsakonas S, Rand MD, Lake RJ (1999) Notch signaling: cell fate control and signal integration in development. Science 284:770-776.

Bessarab DA, Chong SW, Korzh V (2004) Expression of zebrafish six1 during sensory organ development and myogenesis. Dev Dyn 230:781-786.

Bierkamp C, Campos-Ortega JA (1993) A zebrafish homologue of the Drosophila neurogenic gene Notch and its pattern of transcription during early embryogenesis. Mech Dev 43:87-100.

Blader P, Fischer N, Gradwohl G, Guillemot F, Strähle U (1997) The activity of neurogenin 1 is controlled by local cues in the zebrafish embryo. Development 124:4557-4569.

Brooker R, Hozumi K, Lewis J (2006) Notch ligands with contrasting functions: Jagged1 and Delta1 in the mouse inner ear. Development 133:1277-1286.

Cabrera C (1990) Lateral inhibition and cell fate during neurogenesis in Drosophila: the interactions between scute, Notch and Delta. Development 110:733-742.

Calleja M, Renaud O, Usui K, Pistillo D, Morata G, Simpson P (2002) How to pattern an epithelium: lessons from achaete-scute regulation on the notum of Drosophila. Gene 292:1-12.

Chitnis A, Henrique D, Lewis J, Ish-Horowicz D, Kintner C (1995) Primary neurogenesis in Xenopus embryos regulated by a homologue of the Drosophila neurogenic gene Delta. Nature 375:761-766.

Cubas P, de Celis JF, Campuzano S, Modolell J (1991) Proneural clusters of achaete-scute expression and the generation of sensory organs in the Drosophila imaginal wing disc. Genes Dev 5:996-1008.

Daudet N, Lewis J (2005) Two contrasting roles for Notch activity in chick inner ear development: specification of prosensory patches and lateral inhibition of hair-cell differentiation. Development 132:541-551.

de la Pompa JL, Wakeham A, Correia KM, Samper E, Brown S, Aguilera RJ, Nakano T, Honjo T, Mak TW, Rossant J, Conlon RA (1997) Conservation of the Notch signalling pathway in mammalian neurogenesis. Development 124:1139-1148.

Gamba L, Cubedo N, Lutfalla G, Ghysen A, Dambly-Chaudiere C (2010) lef1 controls patterning and proliferation in the posterior lateral line system of zebrafish. Dev Dyn 239:3163-3171.

Ghysen A, Dambly-Chaudière C (2007) The lateral line microcosmos. Genes Dev 21:2118-2130.

Guo M, Jan LY, Jan YN (1996) Control of daughter cell fates during asymmetric division: interaction of Numb and Notch. Neuron 17:27-41.

Haas P, Gilmour D (2006) Chemokine signaling mediates self-organizing tissue migration in the zebrafish lateral line. Dev Cell 10:673-680.

Haddon C, Smithers L, Schneider-Maunoury S, Coche T, Henrique D, Lewis J (1998a) Multiple delta genes and lateral inhibition in zebrafish primary neurogenesis. Development 125:359-370.

Haddon C, Jiang YJ, Smithers L, Lewis J (1998b) Delta-Notch signalling and the patterning of sensory cell differentiation in the zebrafish ear: evidence from the mind bomb mutant. Development 125:4637-4644.

Holley SA, Jülich D, Rauch GJ, Geisler R, Nüsslein-Volhard C (2002) her1 and the notch pathway function within the oscillator mechanism that regulates zebrafish somitogenesis. Development 129:1175-1183.

Itoh M, Chitnis AB (2001) Expression of proneural and neurogenic genes in the zebrafish lateral line primordium correlates with selection of hair cell fate in neuromasts. Mech Dev 102:263-266.

Itoh M, Kim CH, Palardy G, Oda T, Jiang YJ, Maust D, Yeo SY, Lorick K, Wright GJ, Ariza-McNaughton L, Weissman AM, Lewis J, Chandrasekharappa SC, Chitnis AB (2003) Mind bomb is a ubiquitin ligase that is essential for efficient activation of Notch signaling by Delta. Dev Cell 4:67-82.

Kiernan AE, Cordes R, Kopan R, Gossler A, Gridley T (2005) The Notch ligands DLL1 and JAG2 act synergistically to regulate hair cell development in the mammalian inner ear. Development 132:4353-4362.

Kim CH, Bae YK, Yamanaka Y, Yamashita S, Shimizu T, Fujii R, Park HC, Yeo SY, Huh TL, Hibi M, Hirano T (1997) Overexpression of neurogenin induces ectopic expression of HuC in zebrafish. Neurosci Lett 239:113-116.

Koo BK, Lim HS, Song R, Yoon MJ, Yoon KJ, Moon JS, Kim YW, Kwon MC, Yoo KW, Kong MP, Lee J, Chitnis AB, Kim CH, Kong YY (2005) Mind 
bomb 1 is essential for generating functional Notch ligands to activate Notch. Development 132:3459-3470.

Korzh V, Sleptsova I, Liao J, He J, Gong Z (1998) Expression of zebrafish bHLH genes ngnland $n r d$ defines distinct stages of neural differentiation. Dev Dyn 213:92-104.

Kozlowski DJ, Whitfield TT, Hukriede NA, Lam WK, Weinberg ES (2005) The zebrafish dog-eared mutation disrupts eyal, a gene required for cell survival and differentiation in the inner ear and lateral line. Dev Biol 277:27-41.

Laguerre L, Soubiran F, Ghysen A, König N, Dambly-Chaudière C (2005) Cell proliferation in the developing lateral line system of zebrafish embryos. Dev Dyn 233:466-472.

Lai EC, Roegiers F, Qin X, Jan YN, Rubin GM (2005) The ubiquitin ligase Drosophila Mind bomb promotes Notch signaling by regulating the localization and activity of Serrate and Delta. Development 132:2319-2332.

Ma EY, Raible DW (2009) Signaling pathways regulating zebrafish lateral line development. Curr Biol 19:R381-R386.

Matsuda M, Chitnis AB (2010) Atohla expression must be restricted by Notch signaling for effective morphogenesis of the posterior lateral line primordium in zebrafish. Development 137:3477-3487.

Metcalfe WK (1989) Organization and development of the zebrafish posterior lateral line. In: The mechanosensory lateral line neurobiology and evolution (Coombs S, Görner P, Münz H, eds), pp 147-159. New York: Springer.

Millimaki BB, Sweet EM, Dhason MS, Riley BB (2007) Zebrafish atoh1 genes: classic proneural activity in the inner ear and regulation by Fgf and Notch. Development 134:295-305.

Mizoguchi T, Verkade H, Heath JK, Kuroiwa A, Kikuchi Y (2008) Sdf1/ Cxcr4 signaling controls the dorsal migration of endodermal cells during zebrafish gastrulation. Development 135:2521-2529.

Obholzer N, Wolfson S, Trapani JG, Mo W, Nechiporuk A, Busch-Nentwich E, Seiler C, Sidi S, Söllner C, Duncan RN, Boehland A, Nicolson T (2008) Vesicular glutamate transporter 3 is required for synaptic transmission in zebrafish hair cells. J Neurosci 28:2110-2118.

Oka C, Nakano T, Wakeham A, de la Pompa JL, Mori C, Sakai T, Okazaki S, Kawaichi M, Shiota K, Mak TW, Honjo T (1995) Disruption of the mouse RBP-J kappa gene results in early embryonic death. Development 121:3291-3301.

Pandur PD, Moody SA (2000) Xenopus Sixl gene is expressed in neurogenic cranial placodes and maintained in the differentiating lateral lines. Mech Dev 96:253-257.

Radosevic M, Robert-Moreno A, Coolen M, Bally-Cuif L, Alsina B (2011) Her9 represses neurogenic fate downstream of Tbx1 and retinoic acid signaling in the inner ear. Development 138:397-408.

Raft S, Koundakjian EJ, Quinones H, Jayasena CS, Goodrich LV, Johnson JE, Segil N, Groves AK (2007) Cross-regulation of Ngn1 and Math1 coor- dinates the production of neurons and sensory hair cells during inner ear development. Development 134:4405-4415.

Sahly I, Andermann P, Petit C (1999) The zebrafish eyal gene and its expression pattern during embryogenesis. Dev Genes Evol 209:399-410.

Sarrazin AF, Nuñez VA, Sapède D, Tassin V, Dambly-Chaudière C, Ghysen A (2010) Origin and early development of the posterior lateral line system of zebrafish. J Neurosci 30:8234-8244.

Satoh T, Fekete DM (2005) Clonal analysis of the relationships between mechanosensory cells and the neurons that innervate them in the chicken ear. Development 132:1687-1697.

Scheer N, Riedl I, Warren JT, Kuwada JY, Campos-Ortega JA (2002) A quantitative analysis of the kinetics of Gal4 activator and effector gene expression in the zebrafish. Mech Dev 112:9-14.

Schlosser G (2006) Induction and specification of cranial placodes. Dev Biol 294:303-351.

Schweisguth F, Posakony JW (1992) Suppressor of Hairless, the Drosophila homolog of the mouse recombination signal-binding protein gene, controls sensory organ cell fates. Cell 69:1199-1212.

Sieger D, Tautz D, Gajewski M (2003) The role of Suppressor of Hairless in Notch mediated signalling during zebrafish somitogenesis. Mech Dev 120:1083-1094.

Streit A (2007) The preplacodal region: an ectodermal domain with multipotential progenitors that contribute to sense organs and cranial sensory ganglia. Int J Dev Biol 51:447-4461.

Subach FV, Patterson GH, Renz M, Lippincott-Schwartz J, Verkhusha VV (2010) Bright monomeric photoactivatable red fluorescent protein for two-color super-resolution sptPALM of live cells. J Am Chem Soc 132:6481-6491.

Tsutsumi M, Itoh M (2007) Novel transcript nort is a downstream target gene of the Notch signaling pathway in zebrafish. Gene Expr Patterns 7:227-232.

Yamamoto M, Morita R, Mizoguchi T, Matsuo H, Isoda M, Ishitani T, Chitnis AB, Matsumoto K, Crump JG, Hozumi K, Yonemura S, Kawakami K, Itoh M (2010) Mib-Jag1-Notch signalling regulates patterning and structural roles of the notochord by controlling cell-fate decisions. Development 137:2527-2537.

Zeng C, Younger-Shepherd S, Jan LY, Jan YN (1998) Delta and Serrate are redundant Notch ligands required for asymmetric cell divisions within the Drosophila sensory organ lineage. Genes Dev 12:1086-1091.

Zheng W, Huang L, Wei ZB, Silvius D, Tang B, Xu PX (2003) The role of Six 1 in mammalian auditory system development. Development 130: 3989-4000.

Zou D, Silvius D, Fritzsch B, Xu PX (2004) Eyal and Six1 are essential for early steps of sensory neurogenesis in mammalian cranial placodes. Development 131:5561-5572. 